\title{
Actividad antimicrobiana del extracto hidroalcohólico de Piper angustifolium (Piperaceae) sobre Proteus mirabilis
}

\section{Antimicrobial activity of the hydroalcoholic extract of Piper angustifolium (Piperaceae) on Proteus mirabilis}

\author{
(iD) Gina Zavaleta-Espejo ${ }^{\natural}$, (iD Cristina Zavaleta-Castro, (iD José Saldaña-Jiménez, \\ (iD) Anthony Aguilar-Sánchez
}

Universidad Nacional de Trujillo. Trujillo, Perú

\begin{tabular}{llll}
\hline Recibido: 14/04/2018 & Revisado: 26/04/2019 & Aceptado: 14/05/2019 & Publicado: 30/06/2019 \\
\hline
\end{tabular}

\section{RESUMEN}

El objetivo de la presente investigación fue determinar la actividad antimicrobiana de las diferentes concentraciones del extracto hidroalcohólico de hojas de Piper angustifolium "matico" sobre el crecimiento de Proteus mirabilis en condiciones de laboratorio. Para la obtención del extracto hidroalcohólico de hojas de P. angustifolium se empleó un equipo Soxhlet con alcohol etílico al $95 \%$, se hicieron diluciones con Tween 80 (10, 15, 20, 25 y 30 mg/ml).

Para evaluar la actividad antimicrobiana del extracto se empleó el método Kirby-Bauer con discos de papel Watman $\mathrm{N}^{\circ} 4$ de 0,7 mm, empleándose cinco discos para cada una de las concentraciones más un control positivo (Ciprofloxacina ${ }^{\circledR}$ ) y un control negativo (Tween 80), las placas fueron incubadas a $37^{\circ} \mathrm{C}$ durante 24 horas, posteriormente fueron medidos los diámetros $(\mathrm{mm})$ de los halos de inhibición.

Se obtuvo el mayor halo de inhibición $(18,7 \mathrm{~mm}$ ) a la mayor concentración, encontrando un efecto directamente proporcional entre la concentración del extracto y la actividad antimicrobiana. Los resultados evidenciaron diferencias significativas entre las diferentes concentraciones utilizadas.

Palabras clave: Extracto hidroalcohólico; Piper angustifolium; Proteus mirabilis; matico; inhibitorio.

\section{ABSTRACT}

The aim of the present investigation was to determine the antimicrobial activity of different concentrations of hydroalcoholic extract of Piper angustifolium "matico" leaves on the growth of Proteus mirabilis under laboratory conditions. To obtain the hydroalcoholic extract of P. angustifolium leaves, Soxhlet equipment with $95 \%$ ethyl alcohol was used; dilutions were made with Tween 80 (10, 15, 20, 25 and $30 \mathrm{mg} / \mathrm{ml})$.

To evaluate the microbial activity of the extract, the Kirby-Bauer method with $0.7 \mathrm{~mm}$ Watman No. 4 paper discs was used, using five discs for each of the concentration, using ciprofloxacin as a positive control and a negative control (Tween 80), the plates were incubated at $37^{\circ} \mathrm{C}$ for 24 hours, after which time the diameters $(\mathrm{mm})$ of the inhibition zones were measured.

The highest inhibition halo $(18.7 \mathrm{~mm})$ was obtained at the highest concentration, finding a directly proportional effect between the concentration of the extract and the antimicrobial 
activity. The results showed significant differences between the different concentrations used.

Keywords: Hydroalcoholic extract; Piper angustifolium; Proteus mirabilis; matico; inhibitory.

\section{INTRODUCCIÓN}

El Perú cuenta con una gran cantidad de plantas medicinales, las mismas que se convierten en la principal herramienta para tratar diversas enfermedades (Ruiz y Roque, 2009).

La Organización Mundial de Salud (OMS) plantea que las diversas especies vegetales son una de las mejores fuentes de fármacos para la humanidad (Zuta, 2014).

Las piperáceas contienen principios activos que se utilizan como agentes terapéuticos en el descubrimiento de fármacos (Mgbeahuruike et al., 2017). La familia Piperaceae consta de 8 géneros y 3000 especies distribuidas en varias regiones del planeta (Oyemitan, 2017).

Las plantas del género Piper posee aceites esenciales, alcaloides, lignanos, terpenoides, flavonoides, saponinas entre otros (Pecková et al., 2017) son ampliamente utilizados en la medicina tradicional para el tratamiento de diferentes enfermedades (Jiménez, 2011).

Las infecciones del tracto urinario comúnmente ocasionadas por ciertas bacterias que producen la mayoría de infecciones urinarias con frecuencia como: Escherichia coli, Staphylococcus sp, Enterococcus sp, Klebsiella pneumoniae, Pseudomona aeruginosa, Enterobacter $s p$, Proteus mirabilis (Varela, 2008).

El género Proteus, está muy difundido en la naturaleza, encontrándose en el suelo, agua, aguas servidas, materiales de animales en descomposición, tracto intestinal del hombre, entre otros.

Es un patógeno oportunista perteneciente a la familia Enterobacteriaceae, tiene alta motilidad y puede generar diversos tipos de enfermedades infecciosas del tracto urogenital humano (Schaffer y Pearson, 2015).

Dentro del género Proteus las especies más importantes son: Proteus mirabilis y Proteus vulgaris. P. mirabilis es productora de ureasa, factor de patogenicidad en la producción de infecciones urinarias, esta enzima desdobla a la urea en amoníaco y dióxido de carbono.

El amoníaco es tóxico para las células produciendo irritación del epitelio urinario, desencadenando una reacción inflamatoria. La alcalinidad generada por la orina predispone a la litiasis urinaria (Chalán, 2014).

En la actualidad los antibióticos actúan interfiriendo en algún mecanismo específico del metabolismo celular para inhibir el crecimiento del microorganismo o destruirlo. Para mantener la especie, las bacterias han desarrollado resistencia a la acción de los antibióticos (Rice, 2018).

Recientemente se han reportado cepas clínicas de $P$. mirabilis resistentes a antibióticos betalactamicos como el carbapenem constituyendo un riesgo para la humanidad (Leulmi et al., 2019). 
El aumento de la resistencia antimicrobiana a los antibióticos es un problema de gran transcendencia en las últimas décadas, por lo tanto, surge la necesidad de encontrar nuevos antimicrobianos; por ello, la presente investigación tiene por objetivo determinar la actividad antimicrobiana de las diferentes concentraciones del extracto hidroalcohólico de hojas de Piper angustifolium "matico" sobre el crecimiento de Proteus mirabilis en condiciones de laboratorio.

\section{MATERIAL Y MÉTODOS \\ Material biológico}

Las hojas de Piper angustifolium "matico", se recolectaron en la provincia de Sánchez Carrión, distrito de Huamachuco.

\section{Microorganismo}

Proteus mirabilis fue donado por el laboratorio referencial de La Libertad.

\section{Recolección y procesamiento del material biológico}

Las hojas de $P$. angustifolium fueron depositadas en bolsas de malla, luego se procedió a identificar la especie de acuerdo con las directrices de la OMS sobre buenas prácticas agrícolas y de recolección de plantas medicinales (Organización Mundial de la Salud, 2003).

Posteriormente se lavó las hojas en agua con lejía al 1 \% secándose en estufa con circulación $27^{\circ} \mathrm{C}$ durante 24 horas.

Las hojas secas fueron obtenidas en un molino casero hasta obtener polvo.

El producto se almacenó en frascos de vidrio ámbar. Este material se utilizó para procesos de extracción (Calle y Ferreira, 2006).

\section{Preparación de los extractos}

Secolocó $38 \mathrm{~g}$ de matico en un equipo Soxhlet, para que los principios activos sean extraídos se usó una solución hidroalcohólica al $95 \%$ por 2 horas, posteriormente, lo filtrado se colocó en una placa de Petri, procediéndose a secar con ventilador a temperatura menor a $40{ }^{\circ} \mathrm{C}$, el secado duró aproximadamente 3 horas, lo obtenido del secado se pesó y se diluyó en $10 \mathrm{ml}$ de Tween 80 (Sigma-Aldrich, EEUU) obteniéndose la solución madre (100 $\%)$.

A partir de esta se prepararon las diferentes diluciones $(10,15,20,25$ y $30 \mathrm{mg} / \mathrm{mL})$.

\section{Reactivación de la cepa de $P$. mirabilis}

Se empleó la cepa P. mirabilis por ser un agente patogénico común en infecciones de las vías urinarias. Para la reactivación se utilizó agar nutritivo sembrándose a esta bacteria mediante la técnica de siembra en estría, a partir de esta se preparó el inóculo.

\section{Preparación del inóculo}

Una vez obtenido las colonias de P. mirabilis, se tomó con un asa de 1 a 2 colonias con las mismas características morfológicas y se inoculó en un tubo con solución salina fisiológica hasta que alcance la turbidez del tubo $N^{\circ}$ 0,5 en la escala de McFarland (1x108) (Sacsaquispe y Velásquez, 2002).

\section{Preparación de los discos con el extracto hidroalcohólico de $\mathrm{P}$. angustifolium}

Se empleó el método recomendado por el Sub Comité de Ensayos de Susceptibilidad del NCCLS (National Committee for Clinical Laboratory Standards), basado en el procedimiento descrito originalmente por Kirby-Bauer.

Se utilizaron discos de papel Watman $\mathrm{N}^{\circ} 4$ de $7 \mathrm{~mm}$ de diámetro en los cuales se colocó 
$30 \mu \mathrm{l}$ de cada una de las concentraciones del extracto, luego fueron secados en estufa a $30^{\circ} \mathrm{C}$.

\section{Siembra del inóculo de $P$. mirabilis}

A partir del inóculo de P. mirabilis a la concentración del tubo N 0,5 de MacFarland se sembró $0,1 \mathrm{ml}$ en cada placa.

Luego, se colocaron 5 discos por cada placa: tres discos con una misma concentración, más un control positivo (disco del antibiótico Ciprofloxacina) y un control negativo (disco con Tween 80) sobre la superficie de las placas sembradas, las mismas que fueron incubadas a $37{ }^{\circ} \mathrm{C}$ durante 24 horas, transcurrido este tiempo, se midieron los diámetros $(\mathrm{mm})$ de los halos de inhibición.

\section{Evaluación de los tratamientos}

El efecto de las concentraciones del extracto hidroalcohólico de hojas de $P$. angustifolium fue determinado por el diámetro de los halos de inhibición que se obtuvieron por ausencia de crecimiento bacteriano alrededor del disco con el tratamiento colocado en cada placa. Para esto se fijaron tres categorías: sensible (S), intermedia (I) y resistentes (R) (Sacsaquispe y Velásquez, 2002).

Las interpretaciones por regla general, fueron las siguientes: un diámetro de inhibición de 20 a 35 mm es indicativo de una cepa altamente sensible al tratamiento, mientras que diámetros de zona de inhibición entre $16 \mathrm{~mm}$ y $19 \mathrm{~mm}$ se considera intermedio y finalmente diámetros de inhibición inferiores a $15 \mathrm{~mm}$ son presentadas por las cepas resistentes.

\section{Análisis estadístico}

Con los datos obtenidos se estimaron los estimadores paramétricos, así mismo, se aplicó el análisis de varianza y la prueba de comparación múltiple de Tukey $(p \leq 0,05)$ para determinar diferencias y/o semejanzas significativas entre los tratamientos (concentración del extracto).

Se utilizó el programa INFOSTAT (versión libre para estudiantes) 2018.

\section{RESULTADOS Y DISCUSIÓN}

Con relación a los resultados obtenidos en la Figura 1 podemos observar que el diámetro de los halos de inhibición formados por el extracto hidroalcohólico de las hojas de P. angustifolium fueron aumentando conforme se incrementó la concentración del extracto, obteniendo un halo de inhibición de hasta $10 \mathrm{~mm}$ a la menor concentración $(10 \mathrm{mg} / \mathrm{ml})$ y de hasta $18,7 \mathrm{~mm}$ a la mayor concentración (30 $\mathrm{mg} / \mathrm{ml})$; con respecto al grupo control positivo (Ciprofloxanina) con 29,3 $\mathrm{mm}$ y el control negativo que no tuvo ningún efecto, la acción antibacteriana probablemente se debe principalmente a la presencia los diferentes principios activos que han sido reportados en el extracto etanólico del género Piper tales como saponinas, taninos, alcaloides, flavonoides, aceites entre otros (Arroyo et al., 2012).

Los principios activos presentes en las hojas de $P$. angustifolium que fueron extraídos con el solvente hidroalcohólico al $95 \%$ mostraron una actividad antimicrobiana contra $P$. mirabilis.

Es preciso señalar que los diferentes solventes orgánicos como etanol, metanol, cloroformo, etc., usados en la extracción son muy importantes puesto que existen diferencias a nivel de la obtención de los diversos principios activos (Arroyo et al., 2011). 


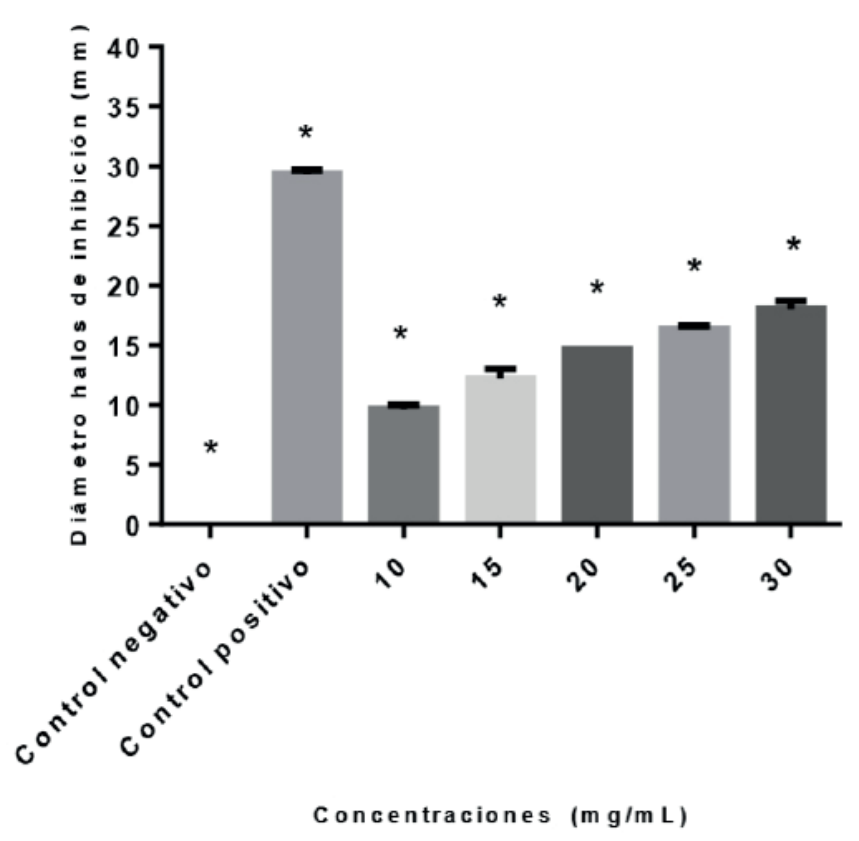

Figura 1. Diámetros promedio (mm) de los halos de inhibición de las concentraciones del extracto hidroalcohólico al 95 \% de Piper angustifolium sobre el crecimiento de P. mirabilis a las 24 horas de incubación.

* Existen diferencias significativas con un $p \leq 0,05$.

Se han realizado estudios utilizando el extracto hidroalcohólico de P. angustifolium mostrando una alta actividad antibacteriana contra Helycobacter pilori obteniendo halos de inhibición de 16 a 20 mm de diámetro (Claros et al., 2007); investigaciones reportaron alta eficacia de diferentes extractos del género Piper contra bacterias Gram negativas y positivas (Mateen et al., 2010; Akthar et al., 2014); es importante recalcar que se ha mostrado el efecto de otras especie del género Piper sobre el crecimiento de P. mirabilis, cuyos resultados difieren en los encontrados en la presente investigación, puesto que utilizaron diferentes tipos de solventes para la extracción de los principios activos, así como los lugares de procedencia de la planta, los órganos de la planta y el tipo de cepa entre otros (Guerrini et al., 2009; Undensi et al, 2012).
Se ha demostrado que los flavonoides del género Piper presentan una buena inhibición sobre las bacterias Gram negativas como por ejemplo $H$. pylori el cual se debería a la hiperacidificación en la interfase de la membrana plasmática de los microorganismos, o la acidificación intracelular, que resulta en la inhibición de la bomba $\mathrm{H}+\mathrm{K}+$ ATPasa necesaria para la síntesis de ATP y esto puede estar relacionado con la inactivación de enzimas celulares que causan cambios en la permeabilidad de la membrana de H. pylori (Arroyo et al., 2013), todo ello afectaría las reacciones en cadena de las bacterias, culminando con una lisis bacteriana; por consiguiente es probable que un mecanismo similar se presente en $P$. mirabilis, puesto que el género Piper presenta una alta cantidad de flavonoide en sus hojas (Soto, 2015). 
Todos los estudios encontrados corroboran lo hallado en esta investigación, donde $P$. angustifoilum "matico" tiene actividad antimicrobiana contra $P$. mirabilis, según el análisis estadístico existen diferencias significativas (Figura 1) entre las diferentes concentraciones utilizadas contra el microorganismo ensayado; sin embargo, a pesar de que existen diversos trabajos sobre la acción antimicrobiana de P. anfustifolium, se debería evaluar el efecto in vivo para así mejorar la eficacia del extracto sin causar ningún daño colateral en las personas.

En la actualidad, la resistencia de Proteus mirabilis a los antimicrobianos farmacéuticos disponibles tiende a incrementarse, por ende se mantiene la búsqueda de nuevos productos que se conviertan en alternativas terapéuticas para controlar las infecciones y superar los problemas de resistencia bacteriana.

Al respecto, García (2006) recomienda el uso de extractos vegetales y aceites esenciales como alternativa importante para el tratamiento de enfermedades bacterianas, debido a que ellas no han desarrollado mecanismos de resistencia en contra de los principios activos de las plantas (Zuta, 2014).

Las especies de Piper contienen alcaloides y otros compuestos bioactivos eso podría ayudar a combatir las infecciones y resolver el problema de aumentar resistencia microbiana a los antibióticos (Vadlapudi y Kaladhar, 2012).

El aislamiento de compuestos bioactivos de estas plantas podría ayudar a identificar nuevos compuestos y podría conducir al desarrollo de nuevos antibióticos naturales.

\section{CONCLUSIONES}

Las diferentes concentraciones del extracto hidroalcohólico al 95 \% de hojas de Piper angustifolium "matico", presentan actividad antimicrobiana sobre Proteus mirabilis en condiciones de laboratorio, observándose que a la concentración de $30 \mathrm{mg} / \mathrm{ml}$ se obtiene el mayor halo de inhibición.

\section{REFERENCIAS BIBLIOGRÁFICAS}

Akthar, S., Birhanu, G., y Demisse, S. (2014). Antimicrobial activity of Piper nigrum L. and Cassia didymobotyra L. leaf extract on selected food borne pathogens, 4(2),911919.

Arroyo, J., Bonilla, P., Moreno-Exebio, L., Ronceros, G., Tomas, G., Huamán, J., Raez, E., Quino, M., y Rodriguez-Calzado, J. (2013). Efecto gastroprotector y antisecretor de un fitofármaco de hojas de matico (Piper aduncum). Rev Peru Med Exp Salud Pública, 30(4), 608-615.

Arroyo, J., Bonilla, P., Tomás, G., y Huamán, J. (2011). Estudio fitoquímico del extracto etanólico y de las fracciones de las hojas de Piper aduncum "matico". Rev. Per. Quím. Ing. Quím, 14(1-2), 62-67.

Arroyo, J., Hañari, R., Tinco, A., Baca, D., Domínguez, L. y Buendía, J. (2012). Efecto antihipertensivo del extracto de Piper aduncum "matico" sobre la hipertensión inducida por L-NAME en ratones. Anales de la Facultad de Medicina, 73(4), 275-280.

Calle, J., y Ferreira, S. (1973). Estudio Fitoquímico del aceite escencial de Piper lenticellosum C. DC. Revista Colombiana de Ciencias Químico-Farmacéuticas, 2(3). 81125.

Chalán, L. (2014). Resistencia bacteriana en bacilos Gram negativos de cultivos aislados de muestras clínicas en pacientes hospitalizados del Hospital "Manuel Ygnacio Monteros" 
durante el periodo octubre - noviembre 2013. (Tesis de pregrado). Universidad Técnica Particular de Loja, Loja, Ecuador.

Claros, M., Bilbao, P., Damiani, E., Gonzáles, E., Estensoro, M., y Alvarez M. (2007). Actividad anti-Helicobacter pylori de Plantago major, Clinopodium bolivianum, Calendula officinalis y Piper angustifolium por el método de difusión de disco. BIOFARBO, 15(1), 37-42.

García, C. (2006). Actividad Antibacteriana de extractos vegetales en cepas hospitalarias de Staphylococcus aureus con resistencia múltiple. (Tesis de doctorado). Universidad Autonoma Agraria Antonio Narro, Torreón, Coahuilla, México.

Guerrini, A., Sacchetti, G., Rossi, D., Paganetto, G., Muzzoli, M., Andreotti, E., Tognolini, M., Maldonado, ME., y Bruni, E. (2009). Bioactivities of Piper aduncum L. and Piper obliquum Ruiz and Pavon (Piperaceae) essential oils from Eastern Ecuador. Environmental Toxicology and Pharmacology, 27: 39-48.

Jiménez, A., Pillco, A., Flores, N., Gonzáles, E., y Bermejo, P. (2011). Evaluación genotóxica del aceite esencial y el extracto etanólico de Piper elongatum Vahl. BIOFARBO, 19(2), 1320.

Leulmi, Z., Kandoulil, C., Mihoubi, I., Benlabed, K., Lezzar, A., y Rolain, J. (2019). First report of blaOXA-24 carbapenemase gene, arm methyltransferase and aac(6')-lb-cr among multidrug-resistant clinical isolates of Proteus mirabilis in Algeria. Journal of Global Antimicrobial Resistance, 16: 1285-129.

Mateen, A., Ahmed, P., Gupta V., Ahmed, M., Uddin, A., Shamshul, A., y Janardhan, K. (2010). A Study of Antimicrobial Activity of Few Medicinally Important Herbal Single
Drugs Extracted in Ethanol, Methanol and Aqueous Solvents. Phcog.Net, 2(10), 351-356.

Mgbeahuruike, E., Yrjönen, T., Vuorela, H., y Holm. Y. (2017). Bioactive compounds from medicinal plants: Focus on Piper species. South African Journal of Botany 112, 54-69.

Organización Mundial de Salud. (2003). Directrices de la OMS buenas prácticas agrícolas y de recolección (BPAR) de plantas medicinales. Ginebra.

Oyemitan, I. (2017). African Medicinal Spices of Genus Piper. Medicinal Spices and Vegetables from Africa. http://dx.doi. org/10.1016/B978-0-12-809286-6.00027-3

Pecková, R., Doležal, K., Sak, B., Květoňová, D., Kváč, M., Nurcahyo, W., y Foitová, I. (2017). Effect of Piper betle on Giardia intestinalis infection in vivo. Experimental Parasitology, 184, 39-45.

Rice, L. (2018). Antimicrobial Stewardship and Antimicrobial Resistance. Medical Clinics of North America, 102(5), 805-818.

Ruiz, Q., y Roque, A. 2009. Actividad Antimicrobiana de cuatro plantas del NorOriente Peruano. Ciencia e Investigación 12(1): 41-47.

Sacsaquispe, C., y Velásquez, P. (2002). Manual de Procedimientos para la Prueba de Sensibilidad Antimicrobiana por el Método de Disco Difusión. Lima, Perú. Instituto Nacional de Salud.

Schaffer, J., y Pearson, M. (2015). Proteus mirabilis and Urinary Tract Infections. Microbiol Spectrum 3(5), 1-39.

Soto, M. (2015). Estudio fitoquímico y cuantificación de flavonoides totales de las 
hojas de Piper peltatum L. y Piper aduncum L. procedentes de la región Amazonas. Scientia, 7(2), 135-140.

Udensi, E., Odom, T., y Dike, C. (2012). Comparative Studies of Ginger (Zingiber officinale) and West African Black Pepper (Piper guineense) Extracts at Different Concentrations on the Microbial Quality of Soymilk and Kunun-zaki. Nigerian Food Journal, 30(2), 38-43.

Vadlapudi, V., y Kaladhar, D. (2012). Phytochemical evaluation and molecular characterization of some important medicinal plants. Asian Pacific Journal of Tropical Disease (2012) S26-S32.

Varela, C. (2008). Comparación de la resistencia al tratamiento de infecciones urinarias no complicadas a nivel internacional, con historias clínicas del servicio de urgencias del hospital San Ignacio del año 2007. Pontificia Universidad Javeriana, Bogota, Colombia.

Zuta, A. (2014). Actividad antibacteriana in vitro de extractos de Piper angustifolium (matico) y Matricaria chamomilla (manzanilla) en cepas de Staphylococcus aureus con resistencia múltiple (tesis de pregrado). Universidad Nacional del Callao, Callao, Perú. 